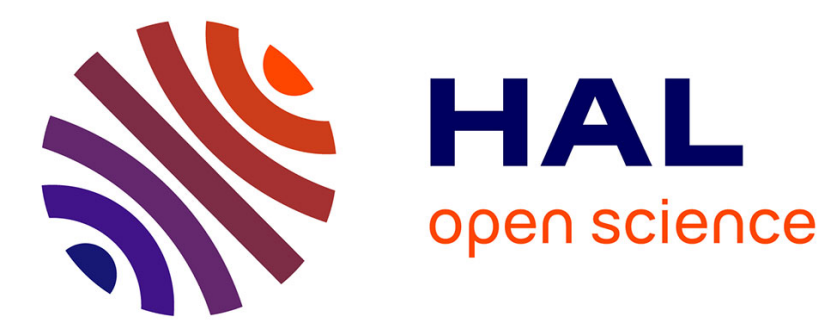

\title{
Évolution de la position du sujet en français : une approche constructionnelle
}

Sophie Prévost

\section{To cite this version:}

Sophie Prévost. Évolution de la position du sujet en français : une approche constructionnelle. Langue française, 2021, N² 209 (1), pp.41-62. 10.3917/lf.209.0041 . hal-03506475

\section{HAL Id: hal-03506475 \\ https://hal.science/hal-03506475}

Submitted on 3 Jan 2022

HAL is a multi-disciplinary open access archive for the deposit and dissemination of scientific research documents, whether they are published or not. The documents may come from teaching and research institutions in France or abroad, or from public or private research centers.
L'archive ouverte pluridisciplinaire HAL, est destinée au dépôt et à la diffusion de documents scientifiques de niveau recherche, publiés ou non, émanant des établissements d'enseignement et de recherche français ou étrangers, des laboratoires publics ou privés. 


\title{
Evolution de la position du sujet en français : une approche constructionnelle
}

\section{Evolution of the position of subjects in French : A constructional approach}

\author{
Sophie Prévost \\ CNRS, ENS-Université PSL et Université Sorbonne Nouvelle
}

\begin{abstract}
Résumé
La fixation progressive de l'ordre des mots en français a conduit au recul de la postposition du sujet, qui s'est majoritairement fixé devant le verbe. Cet article vise à analyser cette évolution dans une perspective constructionnelle, en rendant compte conjointement des sujets nominaux et pronominaux. Nous posons l'existence de trois constructions, SV, VSnominal et VSpronominal lesquelles forment système et sont caractérisées par des propriétés formelles et pragmatiques différentes. Celles-ci ont connu une série de changements qui a conduit à une reconfiguration progressive de ce système avec une spécialisation croissante de VS.
\end{abstract}

Mots-clés

ordre des mots, position du sujet, grammaticalisation, changements constructionnels, français médiéval

\begin{abstract}
The gradual fixation of word order in French resulted in a decrease of the postposition of subjects, most of them having become progressively fixed in preverbal position. This paper aims to analyze this evolution in a constructional perspective, jointly reporting on nominal and pronominal subjects. We posit the existence of three constructions, SV, VSnominal and VSpronominal, which constitute a system and are characterized by different formal and pragmatic properties. These went through a series of changes that led to a gradual reconfiguration of this system and to an increasing specialization of VS.
\end{abstract}

Keywords

word order, subject position, grammaticalization, constructional changes, medieval French

\section{INRODUCTION}

En français moderne l'ordre des mots est relativement rigide, ce qui se traduit par la prévalence, d'une part des objets nominaux ${ }^{1}$ postverbaux (VO) et d'autre part par celles des sujets préverbaux (SV), et donc, quand Sujet, Verbe et Objet sont exprimés, par celle des séquences SVO. La situation était fort différente en ancien français : la plus grande souplesse de l'ordre des mots autorisait bien davantage les séquences OV (même si VO devient majoritaire dès le XI ${ }^{\mathrm{e}}$ s.; voir Marchello-Nizia 1995, 2020a) de même que les séquences VS, et les six combinaisons de ces trois éléments étaient attestées, bien que dans des proportions fort variables, et avec très

\footnotetext{
${ }^{1}$ Les objets pronominaux ont été très tôt antéposés au verbe (voir Marchello-Nizia 1995 et 2020a).
} 
tôt une préférence pour l'ordre SVO (voir Prévost 2020a, 2020b). On se concentrera ici sur la question de la position du sujet, en laissant de côté celle de son expression (même si cette dernière sera évoquée). D'un point de vue quantitatif, les séquences VS ont connu un recul au fil des siècles, différencié selon le type de sujet. D'un point de vue «qualitatif », les séquences VS ont connu des contraintes croissantes, en ce qui concerne les verbes et les sujets impliqués, mais aussi leurs «contextes » d'occurrence. On peut considérer la situation moderne quasiment acquise à la fin du $\mathrm{XVII}^{\mathrm{e}}$ s., même si, et de manière étonnante, la postposition du sujet nominal se développe à la fin du XVIII ${ }^{\mathrm{e}}$ s. et au XIX ${ }^{\mathrm{e}}$ s. dans quelques contextes qu'elle avait désertés, voire ignorés, en particulier dans certaines subordonnées circonstancielles. Ce mouvement reste néanmoins marginal (voir Prévost et Marchello-Nizia 2020 : 1097-1098).

Décrire et analyser l'évolution de la position du sujet suppose de distinguer sujets nominaux (Snom) et sujets pronominaux, tant les caractéristiques des séquences varient, d'un point de vue quantitatif et qualitatif. Parmi les sujets pronominaux, on n'envisagera ici que les pronoms personnels (Sp), les plus nombreux. Les autres pronoms (démonstratifs, possessifs, indéfinis...) ont conservé au fil des siècles un comportement globalement voisin de celui des sujets nominaux, hormis on, qui acquiert à partir du XVII $\mathrm{e}$ s. le comportement des pronoms personnels.

La question de l'évolution de la position du sujet a été abondamment abordée, depuis des décennies, selon des approches très variées (entre autres : Foulet 1930, Vance 1997, Guimier 1997, Marandin 2003, Fuchs 2006, 2013, Lahousse 2011, Prévost 2001, 2010, 2017, Prévost et Marchello-Nizia 2020), avec, le plus souvent, une focalisation sur les aspects formels ou au contraire «fonctionnels » (sémanticopragmatiques), et, dans le premier cas, une prise en compte limitée du contexte. «Paramètre » certes difficile à formaliser, le contexte apporte néanmoins un éclairage précieux, en particulier pour ce qui distingue les structures avec Snom et celles avec Sp, dont l'évolution présente des caractéristiques différentes, qu'une approche en termes de construction peut dans une certaine mesure traiter de manière unifiée.

Nous formulons l'hypothèse qu'il existe dès les débuts du français trois constructions pour exprimer la relation entre le sujet et le verbe :

- SV (sujet ${ }^{2}$-verbe)

- VSnom (verbe-sujet nominal):

1. Mot <moult> m'a pené <peiné> son mariage. (BeroulTristan, fin XII ${ }^{\mathrm{e}}$ s., v. 126)

- VSp (verbe-sujet pronominal) :

2. - Je, fet il, nel vos dirai mie. / Querez autrui qui le vos die. / - Si ferai ge, quant mialz ne puis. » (TroyesYvain, 1177-81, 5229-31)

'Je, dit-il, ne le vous dirai pas. Cherchez quelqu'un d'autre qui vous le dise. - Je le ferai, quand mieux ne puis (= puisque je ne peux rien faire de mieux)'

\footnotetext{
2 Incluant les Snom et les Sp.
} 
Plus précisément on considérera que [sujet-verbe] est une macro-construction (au sens où l'entendent les grammaires de constructions) sans spécification d'ordre entre $\mathrm{S}$ et $\mathrm{V}$, qui se subdivise en trois méso-constructions (SV, VSnom, VSp), lesquelles forment système et sont caractérisées par des propriétés formelles et fonctionnelles.

Dans un premier temps, nous proposerons un rappel historique de l'évolution de la position du sujet en évoquant brièvement les principales explications qui ont été avancées. Dans un second temps, après avoir précisé la notion de construction pour l'objet ici traité, nous envisagerons les changements constructionnels qui ont affecté les propriétés formelles et/ou fonctionnelles et conduit à une reconfiguration du système.

\section{RAPPEL HISTORIQUE ET EXPLICATIONS PROPOSEES}

Nous rendons tout d'abord compte de la progression quantitative de SV (et partant du recul de VS) dans 35 textes s'échelonnant du tout début du XI ${ }^{e}$ s. à la fin du XVII s., avant de retracer les grandes lignes de l'évolution et les explications qui ont été proposées.

\subsection{Evolution quantitative de la position du sujet}

La progression des sujets en position préverbale est nettement différenciée selon leur nature nominale ou pronominale, comme le montre le tableau 1 ci-dessous ${ }^{3}$.

Plusieurs remarques ressortent de ces données. D'un part, l'antéposition du sujet est majoritaire dans la quasi-totalité des textes dès les débuts du français. D'autre part, la fréquence en position préverbale des Sp est toujours nettement plus élevée que celle de Snom. Enfin, la position préverbale du sujet, quel qu'il soit, est toujours plus élevée en subordonnée qu'en principale/indépendante déclarative, et ce dès les plus anciens textes. Pour des raisons de place nous nous concentrerons dans cette étude sur les déclaratives.

\footnotetext{
${ }^{3}$ Les données proviennent de Prévost et Marchello-Nizia (2020). Certains textes ont été analysés intégralement, d'autres échantillonnés. Les sigles utilisés sont développés dans la bibliographie.
} 
Tableau 1 : Fréquence des Snom et des Sp préverbaux dans les déclaratives et dans les subordonnées du XI ${ }^{e}$ s. au XVII $s$.

\begin{tabular}{|c|c|c|c|c|}
\hline es & $\begin{array}{l}\text { Fréquence de } \\
\text { SnomV en } \\
\text { déclarative }\end{array}$ & $\begin{array}{c}\text { Fréquence de } \\
\text { SpV en } \\
\text { déclarative }\end{array}$ & $\begin{array}{l}\text { Fréquence de } \\
\text { SnomV en } \\
\text { subordonnée }\end{array}$ & $\begin{array}{l}\text { Fréquence de } \\
\text { SpV en } \\
\text { subordonnée }\end{array}$ \\
\hline ca 1000 & $63 \%(73 / 116)$ & $84 \% \quad(31 / 37)$ & $74 \% \quad(20 / 27)$ & $94 \%$ \\
\hline StLegier & $71 \% \quad(30 / 42)$ & $84 \% \quad(16 / 19)$ & $(4 / 5)$ & $100 \%$ \\
\hline StAlexis & $38 \%(50 / 131)$ & $78 \% \quad(28 / 36)$ & $67 \% \quad(20 / 30)$ & $100 \% \quad(48 / 48)$ \\
\hline ca 1100 & $53 \%(139 / 261)$ & $81 \% \quad(51 / 63)$ & $53 \% \quad(17 / 32)$ & $(57 / 57)$ \\
\hline Lapidaire & $87 \%(104 / 120)$ & $94 \%(130 / 138)$ & $91 \% \quad(31 / 34)$ & $(77 / 77)$ \\
\hline Eneas1 & $60 \%$ & $94 \% \quad(44 / 47)$ & $71 \% \quad(39 / 55)$ & $100 \% \quad(85 / 85)$ \\
\hline BeroulTristan & $70 \%$ & $84 \% \quad(58 / 69)$ & $71 \% \quad(34 / 48)$ & $(91 / 92)$ \\
\hline TroyesYvain 1177-1181 & $59 \%(6$ & $72 \% \quad(44 / 61)$ & $75 \% \quad(43 / 57)$ & $98 \%$ \\
\hline Aucassin fin XII ${ }^{\mathrm{e}}$-déb XIII ${ }^{\mathrm{e}}$ & $72 \%(84$ & $83 \%(117 / 141)$ & $87 \% \quad(48 / 55)$ & $99 \%(129 / 130)$ \\
\hline ClariConst. après 1 & $43 \%(52 / 122)$ & $57 \% \quad(39 / 68)$ & $98 \%(128 / 131)$ & $97 \%(211 / 217)$ \\
\hline raal & $49 \%(51$ & $80 \% \quad(90$ & $94 \% \quad(6$ & $98 \%$ \\
\hline RenartDole & $57 \% \quad(72$ & $78 \% \quad(79 /$ & $87 \% \quad(40 / 46)$ & $99 \%$ \\
\hline Beaumanoir & $76 \% \quad(6$ & $71 \% \quad(53 / 75)$ & $98 \%(171 / 174)$ & $99 \%($ \\
\hline Joinville & $67 \% \quad(6$ & $92 \%(144 / 156)$ & $98 \% \quad(78 / 80)$ & $100 \%$ \\
\hline MachautFortune 1341 & $83 \% \quad(60 / 72)$ & $90 \% \quad(103$ & $88 \% \quad(81$ & $100^{\circ}$ \\
\hline gier & $83 \% \quad(59 / 71)$ & $(65 / 77)$ & $97 \% \quad(66 / 68)$ & $100^{\circ}$ \\
\hline ldis & $56 \%$ & $89 \%$ & $71 \%$ & 100 \\
\hline Manières & $66 \%$ & $97 \% \quad(244$ & $88 \%$ & $99 \%$ \\
\hline zeJoies & $63 \%$ & $94 \%$ & $(67 / 68)$ & $99 \%$ \\
\hline Sermon & $55 \% \quad(48$ & $93 \%(115$ & $(63 / 89)$ & $100 \%$ \\
\hline OrléansBallades 1415 & $67 \% \quad(56 / 84)$ & $98 \%(16)$ & $88 \% \quad(43 / 48)$ & $100 \%$ \\
\hline Pathelin & $100 \%(42$ & $90 \%(2$ & $80 \% \quad(12 / 15)$ & $100 \%$ \\
\hline CentNou & $82 \% \quad(75$ & $90 \%$ & $92 \% \quad(89 / 98)$ & $99 \% \quad(23$ \\
\hline rBaignollet 1468 & $71 \% \quad(17$ & $95 \%$ & $(7 / 10)$ & $100 \%$ \\
\hline aynes $1490-1505$ & $46 \%(75 / 162)$ & $89 \%$ & $81 \% \quad(82 / 101)$ & $100 \%(183 / 183)$ \\
\hline 494 & $67 \%(70 / 104)$ & $92 \%(114 /$ & $89 \% \quad(88 / 99)$ & $100 \%(182 / 182)$ \\
\hline les & $73 \%$ & $99 \%(143)$ & $91 \% \quad(51 / 56)$ & $99 \%(249$ \\
\hline ettres 1543 & $100 \%($ & $97 \%(255 / 262)$ & $(87 / 97)$ & $99 \%(372 / 373)$ \\
\hline rdMisères 1563 & $91 \% \quad(85$ & $100 \%(80 / 80)$ & $(84 / 89)$ & $100 \%(56 / 56)$ \\
\hline LéryBresil & $93 \% \quad(53 / 57)$ & $93 \%(168 / 180)$ & $95 \%(106 / 112)$ & $99 \%(296 / 297)$ \\
\hline 1592 & $90 \%(104 / 117)$ & 96\% (186/194) & $84 \% \quad(84 / 100)$ & $100 \%(196 / 196)$ \\
\hline eParvenir 1616 & $96 \%$ & $98 \%(202 / 207)$ & $84 \% \quad(60 / 71)$ & $99 \%(157 / 158)$ \\
\hline 1627 & $98 \% \quad(91 / 93)$ & $98 \%(1$ & $90 \%(96 / 107)$ & 12/315) \\
\hline 537 & $100 \%(19 / 19)$ & $100 \%$ & $90 \%(138 / 153)$ & $100 \%$ \\
\hline RabutinLettres 1672-1692 & $100 \%(60 / 60)$ & $99 \%(276 / 279)$ & $(79 / 80)$ & $100 \%(315 / 315)$ \\
\hline & \multicolumn{4}{|c|}{ Position préverbale de $\mathrm{S}$ : inférieure à $50 \%$} \\
\hline & \multicolumn{4}{|c|}{50 et $80 \%$} \\
\hline
\end{tabular}

Dès les plus anciens textes, la postposition des Sp est marginale. Elle dépasse rarement $20 \%$, et tombe sous la barre des $10 \%$ dans la très grande majorité des textes dès le début du XIV ${ }^{\mathrm{e}} \mathrm{s}$. C'est donc, dès le début, une structure marquée d'un point de vue quantitatif. Rien de tel pour les Snom. Leur antéposition est certes très tôt majoritaire dans la plupart des textes, mais sa progression est loin d'être linéaire, et l'amplitude de variation d'un texte à l'autre est parfois forte, y compris entre 
textes contemporains, comme en témoignent les fréquences de sujets préverbaux dans Aucassin (72\%) et ClariConstantinople (43\%) au début du XIII ${ }^{\mathrm{e}}$ s., ou dans Commynes (46\%) et JehanParis $(67 \%)$ à la fin $\mathrm{du} \mathrm{XV}^{\mathrm{e}}$ s. Durant toute la période médiévale $\left(\mathrm{XI}^{\mathrm{e}}-\mathrm{XV}^{\mathrm{e}} \mathrm{s}\right.$.), la progression des sujets préverbaux est très irrégulière : leur fréquence dans QuinzeJoies (63\%), au début du XV $\mathrm{XV}^{\mathrm{e}} \mathrm{s}$., n'est ainsi guère plus élevée que celle dans Eneas1 (60\%) deux siècles et demi plus tôt, ni même que celle de Passion (63\%) au début $\mathrm{du} \mathrm{XI}^{\mathrm{e}} \mathrm{s}$; dans Commynes la fréquence des sujets préverbaux n'atteint que $46 \%$, avoisinant ainsi celles de ClariConstantinople et de Graal, presque trois siècles plus tôt.

Par ailleurs, certains «paramètres » qui semblent influer la plus ou moins forte expression du sujet (voir Prévost et Marchello-Nizia 2020 : 1057-1070), jusqu'au $\mathrm{XV}^{\mathrm{e}}$ s. pour la forme des textes (vers / prose), jusqu'au milieu du XVI s. pour le mode d'énonciation (récit / discours direct), sont inopérants pour ce qui concerne la position du sujet, quelle que soit sa nature : le sujet n'est pas davantage antéposé en prose ou en discours direct, deux contextes pionniers pour la progression de l'expression du sujet. Ainsi ClariConstantinople, texte en prose du début du XIII ${ }^{\mathrm{e}} \mathrm{s}$, affiche une fréquence de Snom préverbaux (43\%) bien inférieure à celle de RenartDole (57\%), texte quasi contemporain en vers. Le domaine (et le genre) des textes, en partie lié à leur forme, n'est pas davantage discriminant : les textes non littéraires (Lapidaire, BeaumanoirBeauvaisis, Mesnagier) privilégient les Snom préverbaux, mais les textes littéraires ont des comportements très variables. Signalons enfin qu'il n'existe pas de lien direct entre le caractère plus ou moins « avancé » d'un texte en matière d'expression du sujet et en matière de position du sujet : dans Graal, la fréquence d'expression du sujet en déclarative est assez élevée (63\%) au regard des textes contemporains, alors que celle de l'antéposition du sujet est bien plus basse (49\%) que dans la plupart des textes de la même époque. Cela n'exclut pas, comme on le verra plus bas, que la hausse de l'expression du sujet ait pu avoir une influence sur sa position, même si ce n'est pas au sein d'un même texte.

Avant d'aborder les propriétés des différentes structures, nous rappellerons très brièvement les explications qui ont été proposées tant pour la variation positionnelle du sujet qui a prévalu pendant la période médiévale que pour le recul de celle-ci au profit d'une linéarisation SV.

\subsection{Rappel des analyses et explications proposées}

\subsubsection{Explications morpho-syntaxiques et sémantico-pragmatiques}

L'évolution du français depuis le latin se caractérise sur le plan syntaxique par une hiérarchisation croissante des syntagmes et par une rigidification de l'ordre des constituants majeurs de la phrase $\left(\mathrm{S}, \mathrm{V}\right.$ et $\left.\mathrm{O}^{4}\right)$, les deux étant en partie liés. La fixation de la position du sujet devant le verbe est un processus en relation avec, d'une part la fixation de l'objet nominal après le verbe, acquise très tôt (Marchello-

\footnotetext{
4 On laissera ici de côté l'attribut. La question de sa position est abordée dans l'article de Lauwers et Vajnovszki dans ce numéro, et nous renvoyons par ailleurs à Marchello-Nizia 2020b et Prévost 2020 b.
} 
Nizia 1995, 2020a), et d'autre part la systématisation de l'expression du sujet, qui s'étale de la fin du XII ${ }^{\mathrm{e}}$ s. au début du XVII ${ }^{\mathrm{e}}$ s. (Prévost et Marchello-Nizia, 2020 : 1055-1079). Deux spécificités de la langue médiévale ont traditionnellement permis de rendre compte de la souplesse de l'ordre des mots et de la postposition du sujet. Il s'agit d'une part de l'existence d'une déclinaison nominale à deux cas (héritage simplifié du latin), qui permettait de discriminer les fonctions des constituants indépendamment de leur position par rapport au verbe. Il s'agit d'autre part de la contrainte relativement forte qui pèse sur le verbe, qui doit occuper la seconde position de la phrase, la présence devant le verbe d'un élément autre que le sujet entraînant la postposition de ce dernier. Cette double explication, qui a prévalu aussi bien dans des approches très descriptives (Foulet 1930) que dans le cadre de la grammaire générative (Vance 1997), a permis de rendre compte aussi du recul progressif de la postposition du sujet, qui résulterait à la fois de la disparition de la contrainte du verbe en seconde position et du déclin progressif de la déclinaison nominale: la disparition d'indices morphologiques permettant d'identifier la fonction syntaxique des constituants et la possibilité que plusieurs éléments précèdent le verbe auraient conduit à la fixation progressive du sujet en position préverbale (sans que ne disparaisse totalement la possibilité d'occuper une position postverbale, comme on le verra plus loin) ${ }^{5}$.

Cette double explication présente des faiblesses. En effet, d'une part la contrainte du verbe en seconde position n'a jamais été absolue, et l'on trouve en ancien français des séquences SOV ou OSV, dans lesquelles le verbe occupe donc la troisième position (3), ou à l'inverse des exemples dans lesquels il occupe la première position (4):

3. Franc et paien merveilus colps $i$ rendent. (Roland, ca 1100, v. 1397)

'Francs et païens des coups redoutables échangent'

4. Plurent Franceis pur pitet <pitié> de Rollant. (Roland, v. 3120)

D'autre part la postposition du sujet reste encore très fréquente alors que la déclinaison nominale n'est plus strictement respectée, et même après sa disparition (Schøsler 1984 a par ailleurs montré que, outre la déclinaison, plusieurs facteurs permettaient de discriminer les fonctions). De plus, la déclinaison des pronoms personnels ayant toujours été respectée (et l'étant encore : il / le / lui), la variation positionnelle de ces derniers aurait dû être plus importante, et se maintenir ; or ce n'est pas le cas. Ces deux facteurs - verbe en seconde position et existence d'une déclinaison - ne suffisent donc pas à rendre compte de la variation positionnelle du sujet en français médiéval, pas plus que leur disparition ne permet d'expliquer la fixation du sujet devant le verbe (sa postposition restant d'ailleurs possible sous certaines conditions). Sans qu'il faille les rejeter, on ne saurait les dissocier de considérations sémantiques, pragmatiques et informationnelles.

\footnotetext{
5 Pour une présentation plus détaillée des explications résumées ici très brièvement, voir Prévost et Marchello-Nizia 2020, et les références qui s'y trouvent.
} 
En ancien français, l'ordre des mots était organisé pour une large part selon un principe informationnel, formulé en termes de «thème-rhème », le dynamisme communicatif tendant à agencer les éléments des moins informatifs vers les plus informatifs (Firbas 1992), ou en termes de «topique-commentaire » (Lambrecht 1994), ce qui n'excluait pas que la position initiale accueille des éléments focalisés ou rhématiques, avec un effet de mise en relief. Ce principe permet d'expliquer (conjointement à la tendance forte à placer le verbe en seconde position) l'agencement des éléments dans la proposition, et plus spécifiquement la position variable du sujet. Dans la mesure où le sujet correspondait souvent au topique et / ou était porteur d'une faible charge informative, il occupait, plus que d'autres éléments, la position préverbale (c'était particulièrement le cas pour le $\mathrm{Sp}$, topique par excellence, d'où la rareté, dès les premiers textes, de sa postposition ; voir Prévost 2010), et il se serait peu à peu fixé en cette position, laquelle, progressivement aurait été réinterprétée comme celle du sujet, le déclin progressif de la déclinaison ayant concouru à cette fixation du sujet devant le verbe.

La question du lien entre la progression de la position préverbale du sujet et celle de son expression ne peut manquer d'être posée. S'agit-il d'un rapport de cause à effet ? D'une évolution conjointe ? L'examen des données et l'affinement des différentes chronologies permettent d'esquisser un scénario. Il apparaît en effet que la primauté revient à l'expression du sujet, qui connaît une forte hausse dès la fin du XII ${ }^{\mathrm{e}}$ s. (voir Prévost 2017, Prévost et Marchello-Nizia 2020). Or dans la mesure où ce sont les pronoms personnels qui ont profité de cette hausse et qu'ils ont toujours majoritairement occupé la position préverbale, la hausse de l'expression du sujet a de facto entraîné celle des sujets préverbaux. On peut formuler l'hypothèse que, par analogie, les autres sujets (en particulier nominaux, les plus nombreux) aient eu de plus en plus tendance à précéder le verbe. Une telle explication n'est pas exclusive de celles présentées précédemment, la convergence de plusieurs facteurs, y compris d'ordres différents (en l'occurrence phonético-morphologiques, syntaxiques et sémantico-pragmatiques), n'étant pas exceptionnelle dans les évolutions de la langue. Le scénario esquissé trace les grandes lignes du changement, tout en en proposant une explication complexe, mais il laisse dans l'ombre les étapes de celuici, et peine quelque peu à articuler les aspects formels et fonctionnels. Une approche dans le cadre de la grammaticalisation ne résout que partiellement cette difficulté.

\subsubsection{Un cas de grammaticalisation?}

Envisagée au regard des paramètres proposés par Lehmann (1995), l'évolution de $\mathrm{Sp}$ et des structures SpV/VSp présente de nombreux indices de grammaticalisation. Le pronom a en effet connu une baisse de son intégrité («désémantisation » et attrition phonologique), la perte des traits de prédicativité, entraînant celle de l'aptitude qu'il avait en ancien français à être déterminé et coordonné. On observe par ailleurs une baisse de la variabilité paradigmatique, puisque le pronom doit désormais être exprimé, et syntagmatique, puisqu'il doit l'être, majoritairement, en position préverbale. Enfin, le processus de cliticisation progressive du pronom a conduit à une baisse de la portée et à une hausse de la cohésion. On peut par ailleurs considérer que la construction sujet-verbe s'est grammaticalisée, perspective qui permet d'inclure les structures avec Snom (les 
Snom eux-mêmes ne présentant pas les caractéristiques d'évolution des Sp). Il s'est ainsi produit une réinterprétation d'un ordre initialement informationnel (voir 2.2.1.) en un ordre syntaxique «sujet-verbe », ce qui correspond à une fixation de stratégies discursives en des structures morpho-syntaxiques (Givón 1979). La position du pronom, comme celle du nom, est devenu l'indice de la fonction sujet, tandis que la position préverbale est devenue celle du sujet (et non plus celle du topique/thème). Il y a donc eu grammaticalisation de la position du pronom ou du nom, et du contenu de la position préverbale, avec désormais coïncidence entre les deux. Si la réanalyse a joué un rôle important dans le processus général, en particulier pour les Sp, l'analogie (aussi bien comme pensée que comme mécanisme) apparaît comme décisive, le pronom personnel jouant le rôle de pivot du changement entre la progression de l'expression du sujet et celle de l'ordre sujet-verbe (voir 2.2.1 cidessus pour le lien entre expression et position).

Sans remettre en question les approches qui ont été proposées ci-dessus, nous proposons d'esquisser une approche plus globale de la régression de la postposition du sujet, en l'envisageant dans un cadre constructionnel.

\section{UNE APPROCHE CONSTRUCTIONNELLE}

Dans le champ de l'approche diachronique des constructions, on distingue les constructionnalisations, qui correspondent à l'émergence de nouvelles constructions et les changements constructionnels, qui affectent des constructions déjà existantes (Traugott et Trousdale 2013: 22 et 26). C'est des seconds qu'il s'agit ici, dans la mesure où les différentes instanciations de la relation sujet-verbe $(\mathrm{SpV}, \mathrm{SnomV}$, regroupées sous SV, VSnom et VSp) sont attestées des débuts du français à nos jours. Leurs propriétés se sont en revanche modifiées.

\subsection{La notion de construction est-elle pertinente pour [sujet-verbe]?}

Il y a, pour le sujet ici traité, certaines difficultés à circonscrire ce que l'on entend par « construction ».

La première difficulté touche à l'extension de la notion de construction et à la prise en compte du contexte. La notion de construction, loin d'être univoque (voir Legallois 2016), a connu une spécialisation définitionnelle dans le cadre des grammaires de constructions, qui oblige désormais à utiliser ce terme avec prudence, en spécifiant quelle acception - et quelle extension - on lui donne. Comme le soulignent Traugott et Trousdale (2013:31), pendant des décennies le terme a été employé pour référer à un syntagme ou un constituant, ou au contexte syntaxique dans lequel un item grammatical se développe. La distinction entre construction et contexte permet d'éviter un certain flou, mais il convient de préciser l'extension de la notion de contexte. Cette dernière peut recouvrir deux choses : le contexte (qu'il soit linguistique ou extra-linguistique : groupe social, tranche d'âge...) dans lequel s'initie un changement (innovation), la nouvelle construction étant susceptible de se propager ensuite à un nombre croissant de contextes, actualisant ainsi le changement. Mais « contexte » peut aussi s'entendre dans un sens plus restreint (sur cette question voir Smirnova 2015). Dans le cas de la construction [sujet-verbe], les éléments initiaux de la phrase constituent un élément déterminant dans la 
caractérisation des constructions VSnom et VSp, et de leur évolution. On peut dès lors se demander s'ils doivent être intégrés dans leurs propriétés mêmes. Nous le ferons au niveau des «micro-constructions » (voir plus bas). La question se pose aussi quant à la présence ou non d'un objet nominal, mais cette caractéristique peut cependant être encodée (dans une certaine mesure) au niveau du verbe, en spécifiant son caractère transitif ou non.

La seconde difficulté est liée à la notion de sens ('meaning'), au cœur de la définition des constructions ('a form-meaning pairing'), qui ne va pas de soi lorsqu'il s'agit de l'ordre des constituants. On admettra ici que le sens général de la construction [sujet-verbe] correspond à une fonction, celle de dénoter une relation prédicative entre un verbe et son sujet ${ }^{6}$, à laquelle s'ajoutent différents «effets de sens », qui relèvent, plus que du plan strictement sémantique, des plans pragmatique et discursif.

Il reste enfin à préciser le «niveau» des constructions ici envisagé, leur « degré de schématicité ». Nous aurons recours au triplet «macro-construction / méso-construction /micro-construction ${ }^{7}$. La macro-construction correspond au couple [sujet-verbe], sans spécification de la linéarisation de ses éléments. Du point de vue de la forme, elle comporte un sujet et un verbe, non spécifiés. Du point de vue du sens, cette construction a une fonction (cf. supra). Cette macro-construction se subdivise en trois méso-constructions: SV, VSnom et VSp, qui héritent des propriétés générales de [sujet-verbe], avec des restrictions pesant sur le sujet, nominal pour VSnom et pronominal pour VSp, et des effets de sens spécifiques.

\subsection{Une série de changements « formels »}

Nous envisageons successivement les changements touchant aux sujets, aux verbes, aux aspects morpho-syntaxiques des séquences SV et VS, et aux éléments initiaux.

\subsubsection{Les sujets}

Il n'y a guère à dire sur la nature des sujets, constitutive des méso-constructions (Snom ou Sp), hormis le fait que le pronom on (issu du substantif hom 'homme') est passé du statut de pronom indéfini à celui de pronom personnel, comme le prouvent les structures VS dans lesquelles on le rencontre. Celles-ci sont assez diversifiées jusqu'au milieu du XVII ${ }^{\mathrm{e}}$ s., puis se restreignent aux seules structures accueillant des $\mathrm{Sp}$.

Se pose par ailleurs la question, au moins pour les Snom, de la définitude et du statut cognitif du sujet ${ }^{8}$. Pour ce qui est de la première, on observe peu de changements: en ancien français, les sujets définis se rencontrent en position préverbale et postverbale, et cette caractéristique s'est maintenue jusqu'à nos jours.

\footnotetext{
${ }^{6}$ Nous laissons ici de côté les constructions impersonnelles (il pleut, il faut partir, il arrive beaucoup de monde, ...) qui demandent un développement spécifique.

7 Et n'adoptons donc pas la terminologie «schéma / sous-schéma / micro-construction » qui l'a remplacé dans certains travaux (voir Traugott et Trousdale $2013: 16$, note 13).

${ }^{8}$ Le fait de recourir à un pronom personnel suppose que le référent est défini et connu. Cela ne recouvre pas exactement la même chose selon la personne considérée, mais la question n'est pas pertinente pour notre propos.
} 
5. Quant il eurent leur letres, si s'en revint li vesques $<$ l'évêque $>$ au plus tost qu'il peut (ClariConstantinople, ap. 1205, p. 15)

Quant aux sujets indéfinis, ils ont toujours été peu fréquents en position préverbale, la langue privilégiant soit leur position après le verbe, soit le recours à des constructions alternatives, telles que les constructions présentatives ou clivées. Le statut cognitif des Snom est largement lié à leur définitude, qui l'est elle-même à leur codage morpho-syntaxique (voir Prévost 2001: 51-63): on ne trouve que rarement de sujet cognitivement inactif ou non identifiable (voir Ariel 1988) en position préverbale, mais l'on trouve à l'inverse des sujets actifs après le verbe : la relation entre définitude / statut cognitif et position n'est donc pas bi-univoque, et la position postverbale semble ouverte à une diversité de sujets plus grande.

\subsubsection{Les verbes}

Les structures SV ont toujours accueilli tous types de verbe, transitifs et intransitifs. C'est aussi le cas des structures VS durant la période médiévale : aucune restriction ne pèse sur les verbes, tant du point de vue sémantique qu'argumental. On trouve ainsi des verbes intransitifs aussi bien que des verbes transitifs (6-7) et attributifs :

6. Iceste piere usent enchanteur a lur enchauntement, (Lapidaire, mi-XII ${ }^{\mathrm{e}}$, p. 7)

'Cette pierre utilisent les magiciens pour leur tour de magie (= Les magiciens utilisent cette pierre ...)'

7. Quant il furent descendu, si gardent avant, si veoient il une fause posterne (ClariConstantinople, p. 75)

'Quand ils furent descendus, [ils] regardent devant eux, alors voient-ils (= ils voient) une fausse poterne'

Dès le milieu du XIII ${ }^{\mathrm{e}}$ s., les constructions transitives reculent dans VS, au moins avec les Snom, du fait de certains changements plus généraux : la fixation croissante de l'objet nominal après le verbe et la tendance à répartir sujet et objet nominaux de part et d'autre du verbe. On trouve néanmoins encore des constructions transitives avec un objet nominal et ce jusqu'au début du XVI $\mathrm{X}^{\mathrm{e}}$ s., très ponctuellement jusqu'au $\mathrm{XVII}^{\mathrm{e}}$ :

8. Ainsi perdit Nicias l'avantage qu'il avoit nettement gaigné sur les Corinthiens. (Montaigne, 1592, p. 97).

9. Ainsin emporte les bestes leur rage à s'attaquer à la pierre et au fer (Montaigne, p. 22)

Les verbes transitifs se sont néanmoins maintenus dans deux types de constructions spécifiques. Il s'agit d'une part de la construction VSpO : Peut-être acceptera-t-il notre offre, en relation avec certains éléments initiaux (voir cidessous), et d'autre part de la construction VOSnom. Celle-ci semble avoir disparu au XVII s., avant de réapparaître à partir du XIX ${ }^{\mathrm{e}}$ s., dans des écrits à caractère administratif ou juridique, avec une contrainte forte pesant sur le sujet, qui doit être «lourd », tandis que l'objet doit être « léger» (Devront repasser l'examen tous les 
étudiants qui ont raté le contrôle continu (exemple emprunté à Fuchs 2013). Nous reviendrons sur ces deux constructions. Les seules restrictions qui s'observent au fil des siècles avec SV concernent la position de l'objet : SOV, en recul dès le XII ${ }^{\mathrm{e}} \mathrm{s}$. a disparu au XVII ${ }^{\mathrm{e}}$ s. (sauf licence poétique), tandis que OSV, après un parcours atypique (recul, progression) s'observe encore en français moderne mais seulement dans des contextes de focalisation de l'objet (Rien que des pâtes (,) ils mangent làbas.) ou de ce que l'on peut assimiler à une dislocation avec ellipse de l'élément de reprise (le chocolat, les enfants adorent ça) (voir Prévost 2020a et 2020b). Les restrictions relatives au type de verbe ont donc davantage pesé sur les constructions VSp/VSnom.

\subsubsection{Contiguïté accrue entre $S$ et $V$ et au sein du complexe verbal}

Différents changements affectent les constructions SV et VS, qui se traduisent par une contiguïté accrue au sein du complexe verbal ou entre le sujet et le verbe.

Les deux changements majeurs concernent VSnom et $\mathrm{SpV}$. Pour les premières, il s'agit plus spécifiquement des structures avec une forme verbale complexe (temps composé, passif ou auxiliaire modal + infinitif). Depuis les débuts du français, deux constructions sont en concurrence, celle dans laquelle le sujet suit directement le verbe conjugué, qui est donc séparé du participe passé ou de l'infinitif (10) et celle dans laquelle le sujet est postposé à l'ensemble du complexe verbal (11). Ce n'est qu'au milieu du XVI ${ }^{\mathrm{e}} \mathrm{s}$, à l'issue d'une évolution irrégulière et marquée par la variation intertextuelle, que l'usage se stabilise au profit de la structure V-Participe passé /Infinitif-Snom (même si quelques cas sporadiques peuvent subsister ( $A$ celui qui a le plus reçu sera le plus grand compte demandé, Pascal, Pensées, 1658, p. 458) (voir Prévost et Marchello-Nizia 2020 : 1085-1087 pour une description plus détaillée).

10. Atant s'en est Iseut tornee. (BeroulTristan, fin XII ${ }^{\mathrm{e}}, \mathrm{v} .233$ ) 'Alors s'en est tournée Iseut (= Alors Yseut est partie)'

11. Mot m'a pené son mariage. (id., v. 126) 'Beaucoup m'a peiné son mariage (= son mariage m'a beaucoup peiné)'

Il existe en ancien français une différence syntaxique importante selon la position de Sp. En position postverbale, le pronom est presque toujours conjoint au verbe, ne pouvant en être séparé que par un autre pronom conjoint $(l e, l a \text {, en ... })^{9}$, contrainte qui s'est maintenue jusqu'en français moderne. En revanche, en position préverbale, le Sp peut être disjoint du verbe par tout type d'élément, y compris un objet nominal, même si ce dernier cas reste rare :

\footnotetext{
9 Skårup (1975 : 47-50) cite de rares cas où un élément autre qu'un pronom complément conjoint (principalement certains adverbes) peut s'intercaler entre le verbe et le pronom postverbal, ce qu'il explique par les exigences de la versification, ou comme des erreurs de copistes.
} 
12. Ne il le roi ne desfia / Ne li rois droit ne li vea. (Roman de Thèbes, mi-XII' s., v. 7933-7934) 'Et il le roi ne défia pas, et le roi son droit ne lui refusa pas'.

Il peut aussi être coordonné et déterminé, et fonctionner comme un énoncé isolé, sans le support d'un verbe conjugué. Dès la fin du XII ${ }^{\mathrm{e}}$ s., le pronom commence à perdre son autonomie syntaxique, et à partir du XIII ${ }^{\mathrm{e}}$ s. ce sont progressivement les formes compléments $(m o i$, lui,$\ldots)$, accentuées, qui prennent la relève des formes sujets dans ces emplois prédicatifs, mouvement qui s'intensifie en moyen français (Marchello-Nizia 1997 : 230-237, Zink 1997: 129-155, Prévost 2020c). La possibilité de disjoindre le pronom personnel sujet du verbe sort de l'usage à partir du XVII ${ }^{\mathrm{e}}$ s., à l'exception de la formule figée : je, soussigné Luc Dubois, certifie ...

Le dernier changement à signaler, d'une ampleur moindre, concerne la distance qui peut séparer le Snom préverbal de son verbe (le Snom postverbal n'en ayant jamais été très éloigné). Jusqu'au XIV ${ }^{\mathrm{e}} \mathrm{s}$., le sujet, quand il n'est pas juxtaposé au verbe, n'en est séparé que par peu d'éléments, le verbe occupant majoritairement la seconde position. Au fur et à mesure que le verbe « recule» dans la proposition, s'ouvre la possibilité de multiplier les éléments devant le sujet, ou entre le sujet et son verbe, tendance qui atteint son apogée aux $\mathrm{XV}^{\mathrm{e}}$ et $\mathrm{XVI}^{\mathrm{e}}$ s., qu'il s'agisse de groupes nominaux, d'adverbes, de participes ou même de subordonnées circonstancielles, rarement d'arguments du verbe :

13. Édouard, prince de Galles, celuy qui regenta si long temps nostre Guienne, personnage, duquel les conditions et la fortune ont beaucoup de notables parties de grandeur, ayant esté bien fort offencé par les Limosins, et prenant leur ville par force, ne peut etre arresté par les cris du peuple, et des femmes, et enfans abandonnez à la boucherie. (Montaigne, p. 7)

L'usage se restreint au XVII ${ }^{\mathrm{e}}$ s.: il n'est désormais plus possible d'intercaler des arguments entre sujet et verbe, ni même des adverbes portant sur le prédicat. On trouve encore des éléments à valeur circonstancielle mais, dès le milieu du siècle, les groupes insérés tendent à être plus réduits, et ils se rattachent sémantiquement et syntaxiquement au sujet. L'insertion de groupes plus longs suppose désormais une reprise pronominale du Snom.

Parmi les différentes restrictions évoquées jusqu'ici, ce sont principalement celles affectant le verbe (recul des verbes transitifs) et dans une moindre mesure les Snom qui sont susceptibles d'être liées à un changement de "sens » de l'énoncé. Les éléments initiaux (c'est-à-dire qui précédent le sujet et le verbe) ont joué de leur côté un rôle majeur.

\subsubsection{Les éléments initiaux}

\section{VSnom}

Jusqu'au XIII $^{\mathrm{e}}$ s. les éléments qui précèdent VSnom sont très variés, par leur nature et par leur fonction (arguments du verbe, participes passés, infinitifs, éléments à valeur circonstancielle, adverbes ou conjonctions à valeur de connecteur 
temporel ou textuel, ...). Mais dès la fin du XII ${ }^{\mathrm{e}}$ s., et la tendance s'accentue au XV s., cette grande diversité tend à se réduire, au détriment des arguments ${ }^{10}$; les autres compléments du verbe (compléments locatifs, objets indirects, ...), peuvent en revanche toujours occuper la position initiale, en particulier s'ils contiennent un élément anaphorique qui rattache l'énoncé au contexte précédent :

14. Et de là s'engendre par fois la défaillance fortuite, qui surprent les amoureux si hors de saison,... (Montaigne, $\mathrm{p}$. 13)

On observe par conséquent une hausse proportionnelle des circonstants, en particulier ceux établissant un lien avec le contexte précédent; le verbe est généralement pourvu d'un sémantisme ténu, l'apport informationnel étant concentré sur le sujet. Cet usage s'est conservé jusqu'au français moderne. Deux éléments méritent un commentaire spécifique. Il s'agit tout d'abord de l'adverbe $s i$, qui, dans toute l'histoire du français, a toujours été suivi de VS ou du verbe seul et jamais de SV (on trouve en revanche Snom-si-V; voir Marchello-Nizia 1985). Jusqu'à la fin du XII ${ }^{\mathrm{e}}$ s., les séquences si-VSnom restent assez rares (10\% des séquences VSnom dans StAlexis et dans Roland). Elles connaissent une hausse importante à partir du $\mathrm{XIII}^{\mathrm{e}}$ s., et sont dès lors attestées, plus ou moins fréquemment, dans la plupart des textes. En début de phrase, si fonctionne comme un adverbe de liaison; après une subordonnée, il a le rôle d'une ligature, difficile à traduire (voir Marchello-Nizia 1985 pour une étude détaillée) :

15. Quant il eurent leur letres, si s'en revint li vesques $<$ l'évêque $>$ au plus tost qu'il peut. (ClariConstantinople, $\mathrm{p}$. $15)$.

Cette structure ne dépasse guère la prose narrative $d u$ XIII $^{\mathrm{e}} \mathrm{s}$., tandis que la structure sans subordonnée reste bien vivante jusqu'à la fin $d u X V^{e}$ s., avant de reculer dans la seconde moitié du XVI ${ }^{\mathrm{e}} \mathrm{s}$. puis de disparaitre à la fin du XVII ${ }^{\mathrm{e}}$ s., en même temps que l'adverbe lui-même :

16. Ores <alors> estoit celuy chat ung groz noir chat fort vieux et estoit mesme de leens <ici>. Si le fist prendre ledit messire Phelippe. (Vigneulles, 1515, p. 228)

Il faut par ailleurs signaler ce fait étonnant (largement commenté, entre autres par Bergh 1952, Beaulier 1956, Antoine 1958-1962, Marchello-Nizia, 1997 : 415) que constitue le développement à partir du XIII ${ }^{\mathrm{e}}$ s., et surtout du XIV ${ }^{\mathrm{e}}$ s., des séquences $e t$-VSnom (de même que des séquences $e t$-V-on), exceptionnelles dans les plus anciens textes alors qu'elle représentent entre 20 et $40 \%$ des séquences VSnom au $X^{\mathrm{e}}$ s. :

17. Et le gallant fera auxi <aussi> d'autre part tant de services et faire des pastez <pâtés> et des tartres qu'ilz mengeront

\footnotetext{
${ }^{10}$ L'attribut s'est bien mieux maintenu, nécessitant cependant progressivement la présence d'un sujet lourd, à l'exception de tel, qui impose d'ailleurs une séquence VSnom : telle est la question versus *la question est telle. Voir Lauwers et Vajnovszki dans ce numéro.
} 
ensembles, et paiera tout le bon homme... (QuinzeJoies, 1400, p. 113).

Et a probablement été perçu, dans certains cas, comme dépassant sa simple valeur de coordonnant au profit d'une valeur adverbiale (c'était un adverbe en latin), et occupant une position pleine devant le verbe. De fait, il tend souvent à introduire des énoncés exprimant une conséquence ou un ajout informatif, et/ou présentant une forme de redondance avec ce qui précède (voir Prévost 2001). Tout en tendant à renforcer le lien entre les deux propositions, ces séquences ont fini par devenir un effet de style, en particulier dans les chroniques. Après une hausse notable jusqu'à la fin du $X^{\mathrm{e}}$ s., et-VSnom se stabilise au milieu du XVI ${ }^{\mathrm{e}}$ s., puis régresse, et sort de l'usage à la fin du XVII ${ }^{\mathrm{e}} \mathrm{s}$. $^{11}$.

Jusqu'au milieu du $\mathrm{XVI}^{\mathrm{e}}$ s., de nombreux adverbes sont encore suivis de VSnom, qu'ils aient une valeur spatiale, temporelle, de manière, ou même épistémique ou discussive :

18. Ainsi perdit Nicias l'avantage qu'il avoit nettement gaigné sur les Corinthiens. (Montaigne, p. 17)

C'est au cours du XVII ${ }^{\mathrm{e}} \mathrm{s}$. que se réduit leur éventail, au profit des seuls adverbes à valeur spatiale ou temporelle, ou parfois de manière, précédant un verbe généralement intransitif, comme c'est encore le cas aujourd'hui.

Cette évolution est à mettre en relation avec un changement plus général qui affecte la syntaxe du sujet nominal : dès le début du XVII ${ }^{\mathrm{e}}$ s., l'ordre VSnom n'est plus apte à affecter la modalité d'énonciation de l'énoncé (voir Fournier 1998 et 2001), ce qui se traduit par le développement de l'inversion dite complexe au détriment de l'interrogation totale par postposition du Snom au verbe, devenue agrammaticale (*Viendra Paul? versus : Paul viendra-t-il ?). Corollairement, en déclarative, derrière des adverbes discussifs ou épistémiques (c'est-à-dire mettant en suspens la validation de la prédication, voir Guimier 1997), se développent pareillement des structures avec inversion complexe :

19. Aussi les livres sont ils en si grande quantité que les bons demeurent accablez sous les mauvais. (SorelBerger, 1627, p. 14).

\section{$\boldsymbol{V S p}$}

Les éléments initiaux qui précèdent VSp sont pareillement diversifiés jusqu'au $\mathrm{XIV}^{\mathrm{e}}$ s. : arguments du verbe (comprenant souvent un élément anaphorique), circonstants, ...mais aussi des adverbes à caractère logico-pragmatique, bien que ceux-ci restent encore peu fréquents :

20. Sire, fet mes sires Gauvains, encor $\boldsymbol{i}$ a il autre chose que vos ne savez mie. (Graal, ca 1225, p. 163c) 'Seigneur, dit monseigneur Gauvain, encore y a-t-il (= il y a encore) une autre chose que vous ne savez pas'

\footnotetext{
${ }^{11} E t$ reste compatible avec des séquences VSnom, mais celles-ci sont indépendantes de la conjonction : (et) arrivèrent alors tous les invités.
} 
L'adverbe $s i$ est globalement assez rare en ancien français ; parfois doté d'une valeur de liaison, il a le plus souvent une valeur d'opposition ou de concession, ou, dans une réponse, de conformité (voir ex. 2 dans l'introduction). Dans l'ensemble des textes, bon nombre des éléments qui précèdent les séquences VSp établissent un lien avec le contexte précédent, souvent par la présence d'un morphème anaphorique, ou bien de si. La postposition du Sp peut dans ce cas être interprétée comme un moyen de resserrer le lien entre le prédicat et ce qui précède. Mais l'on trouve aussi des éléments initiaux qui marquent une opposition ou un contraste avec le contexte précédent :

21. Ultre cest jurn ne surum plus vivant; / Mais d'une chose vos soi jo ben guarant. (Roland, v. 1520-1521) 'Après ce jour [nous] ne serons plus vivants; mais d'une chose vous suis-je bien guarant'

D'une manière générale, et indépendamment du sémantisme de l'élément initial, il n'est pas rare qu'une séquence VSp soit associée à un effet de contraste (focus contrastif) ou d'opposition, en particulier lorsque l'objet nominal est antéposé au verbe :

22. Sire, fet Lancelot, qui fu cil qui tant a parlé a vos? Son cors ne poi je veoir, mes sa parole ö je bien qui est si laide et si espoantable. (Graal, p. 189a) 'Seigneur, fait Lancelot, qui était celui qui vous a parlé si longtemps? Son corps ne pusje voir, mais sa voix entendis-je bien, qui est si laide et épouvantable'

On ne saurait cependant opposer strictement de ce point de vue les constructions $\mathrm{SpV}$ et VSp. Si une relation de contraste ou d'opposition est souvent associée à VSp, en revanche les éléments assurant un lien avec le contexte précédent sont attestés aussi bien devant SpV que VSp. Il semble néanmoins exister, au sein d'un même texte, des phénomènes de répartition complémentaire des éléments initiaux, qui constituent des micro-systèmes propres à chaque texte (voir Prévost 2010).

A partir du milieu du XIV ${ }^{\mathrm{e}}$ s., les arguments et les éléments qui établissent un lien avec le contexte précédent tendent à reculer tandis que les éléments à valeur logique et/ou pragmatique dénotant une rupture, rares en ancien français, connaissent une forte hausse :

23. Et s'il le savoit bien davant qu'il fust marié, si l'a il oublié. (QuinzeJoies, p. 35)

24. si donna le roy Jehan de moult riches dons au roy et a la royne d'Espaigne, ses beau pere et mere, si <pareillement> fit il aux roys d'Arragon, de Portugal et de Navarre. (JehanParis, 1494, p. 91)

On observe ainsi la présence récurrente de subordonnées hypothétiques ou concessives suivies de si à valeur oppositive (23), qui mettent en œuvre un même processus : la subordonnée laisse prévoir une certaine conclusion, qui n'est finalement pas celle qui advient ; il se produit un retournement argumentatif, avec 
une mise en cause de la force argumentative du premier élément d'un enchaînement, ce qui conduit à la validation d'un énoncé malgré un énoncé précédent qui aurait dû s'y opposer (voir Prévost 2001, 2010). Par ailleurs, dans les constructions en si / aussi faire (le faire ou faire de même / pareillement) (24), l'adverbe traduit la conformité, qui concerne le procès lui-même : associé à un verbe vicaire, si / aussi n'évoque aucun procès particulier, mais l'idée générale de procès, qui ne peut se préciser que par référence au contexte. Adverbe porteur de contingence, il « implique un débat au terme duquel une valeur est finalement retenue » (Guimier 1997). Dans les deux cas, il se produit une mise en balance - et donc en suspens - de la relation prédicative. Un tel processus est à l'œuvre dans bon nombre des énoncés VSp de cette époque.

Le $\mathrm{XVII}^{\mathrm{e}}$ siècle marque un second tournant majeur, avec un mouvement de spécialisation au profit des seuls éléments dénotant une mise en balance de la prédication, et au détriment de ceux dénotant un retournement argumentatif. Bon nombre des éléments qui sont encore tolérés au début du siècle (lors, alors, adonc, à tant, bien, difficilement, or ...) ne le sont plus à la fin du siècle, époque à laquelle la situation moderne est acquise ${ }^{12}$. Désormais la postposition du $\mathrm{Sp}$ ne se rencontre plus guère que lorsqu'un adverbe épistémique ou argumentatif précède le verbe : peut-être, sans doute, probablement, aussi, en vain, à peine, au moins, à peine, encore, toujours.... Ces adverbes ne peuvent précéder VSnom, mais ils peuvent être suivis de SnomVSp. Ils ont en commun de déclencher une mise en balance de la relation prédicative, et de conférer ainsi un caractère non pleinement assertif à l'énoncé ${ }^{13}$ :

25. Quant à la question de Dieu ; oui, peut-être as-tu raison, peut-être faut-il croire pour en parler selon Claudel. (Alain Fournier, Correspondance, 1905-1914, p.61 ; Frantext)

\subsection{La spécialisation progressive de VSnom et de VSp}

\subsubsection{Réduction formelle des constructions}

Les constructions SV n'ont connu au fil des siècles qu'une restriction formelle, celle d'une contiguïté accrue entre le sujet et son verbe, tandis que les constructions VSnom et VSp ont connu des restrictions touchant aux verbes et aux éléments initiaux.

L'évolution de la méso-construction VSnom depuis l'ancien français a conduit à l'existence en français moderne de trois micro-constructions, le verbe occupant la position initiale dans deux d'entre elles. La première («inversion inaccusative »), qui a traversé toute l'histoire du français, implique des verbes intransitifs dénotant l'existence, la survenance ou la disparition (arriver, apparaître, passer, ...), ou bien

\footnotetext{
12 Même si ponctuellement on trouve encore par la suite quelques rémanences de l'usage ancien (Grevisse, $1993:$ : 377).

${ }^{13}$ On rencontre parfois VSp derrière certains autres adverbes : du coup, par conséquent, donc, alors, jamais, d'ailleurs... (Grevisse, $1991: \$ 377$, d) pour lesquels on ne peut arguer d'une mise en balance de la prédication. Nous laissons de côté pour cette étude ces exemples rares, et parfois un peu artificiels.
} 
des verbes pronominaux, ou des tournures passives. Peu de contraintes pèsent sur le sujet, volumineux ou non, qui dénote un référent actif ou au contraire non identifiable (Arrive alors la foule des invités). L'hypothèse est que de tels verbes ne sélectionnent pas d'argument externe (sujet), mais seulement un argument interne (objet), qui se déplace vers la position du sujet dans SV, mais reste dans sa position de base dans VS. La seconde micro-construction («inversion élaborative») implique des sujets volumineux et des formes verbales non transitives complexes ou des constructions transitives dans lesquelles l'ordre est invariablement VOSnom. (voir ex. en 3.2.2.). Elle illustre les différentes forces, et leurs tensions, qui président à l'agencement de l'ordre des mots : d'une part sur un plan strictement syntaxique, la réticence de la langue à disjoindre l'objet direct du verbe, à antéposer l'objet au verbe et à juxtaposer sujet et objet nominaux, d'autre part, sur le plan prosodique et informationnel, la tendance à placer un sujet volumineux et porteur d'information après un groupe verbal comparativement court. Dans la troisième microconstruction, le verbe est nécessairement précédé d'un élément, souvent un syntagme locatif ou temporel, mais aussi certains adverbes de manière (ainsi parlait Zarathoustra) de même que certains attributs adjectivaux ${ }^{14}$.

Dans les trois cas, l'ordre SnomV reste le plus souvent « grammaticalement » possible (avec un déplacement de l'élément initial, le cas échéant, derrière SnomV), bien que plus ou moins incongru sur les plans informationnel et prosodique.

La méso-construction VSp n'est plus quant à elle illustrée que par une seule micro-construction ${ }^{15}$ : «adverbe épistémique $+\mathrm{VSp} »:$ peut-être changera-t-il d'avis.

\subsubsection{Effets de sens}

Il convient de déterminer quelles significations sont associées aux mésoconstructions, SV, VSnom et VSp, voire aux micro-constructions, au-delà de la dénotation d'une relation prédicative. Nous faisons l'hypothèse que SV constitue une construction neutre, non marquée. Elle l'est d'un point de vue quantitatif, puisque dès les premiers textes, elle est très largement majoritaire avec les $\mathrm{Sp}$, et qu'elle devient aussi prévalente, bien que plus tardivement et de manière non linéaire, avec les Snom. "Non marqué » ne signifie pas qu'elle produise les mêmes effets de sens que VSnom et VSp, mais qu'elle n'en produit pas.

VSp a toujours été très marginal, ce qui s'explique aisément dans le cadre d'une organisation de l'ordre des mots selon un principe informationnel (voir 2.2.). La construction présente néanmoins en ancien français une relative diversité, qui ne permet pas de lui associer un effet de sens systématique. En effet, si dès cette époque elle est souvent associée à un effet d'opposition, de contraste, cela n'est pas toujours le cas, et certaines occurrences résistent à l'analyse, sauf à y voir un effet de la contrainte du verbe en seconde position, qui, bien que non absolue, demeure

\footnotetext{
14 Toutefois, dans ce dernier cas, à l'exception de tel (telle est la règle), le sujet nominal doit désormais être déterminé par une relative ou un groupe adjectival (*rares sont les cantons suisses), ou bien se trouver dans un système corrélatif.

${ }^{15}$ ce qui n'empêche pas de maintenir la distinction, d'un point de vue théorique, entre méso-construction et micro-construction.
} 
néanmoins un facteur d'influence. A partir du moyen français apparaît en revanche une tendance nette, largement liée aux éléments initiaux, les énoncés VSp dénotant désormais une opposition ou la validation problématique de la relation prédicative (Guimier 1997, Prévost 2001, 2010), cette dernière valeur s'imposant à partir du $\mathrm{XVII}^{\mathrm{e}}$ s., en relation avec la réduction des éléments précédant VSp. C'est cette même valeur que l'on retrouve dans les structures SnomVSp («inversion complexe ») à partir de cette même époque (voir 3.2.4.).

Le principe informationnel est resté influent sur la position des Snom au fil des siècles, même si les procédés mis en œuvre se sont en partie modifiés (voir 3.2.1). C'est ainsi leur statut de focus informationnel qui explique, dès l'ancien français, la postposition de bon nombre de Snom, en particulier indéfinis, qui dénotent un référent non encore instancié, de même que des Snom qui dénotent un référent connu, mais différent de celui du ou des sujet(s) précédent(s), et nécessitant donc une réactivation (26):

26. Li quens Rollant est muntet el destrer. / Cuntre lui vient sis cumpainz Oliver. (Roland, v. 793)

Le comte Roland est monté sur son destrier: à sa rencontre vient son compagnon Olivier.'

Dès les premiers textes, la postposition du sujet résulte aussi de l'utilisation de la première position pour des effets de mise en relief, en particulier de l'attribut et de l'objet, parfois intensifiés, et qui revêtent une valeur de focus ${ }^{16}$. OVS régresse à la fin $\mathrm{du} \mathrm{XV}^{\mathrm{e}}$ s. et disparaît au XVII ${ }^{\mathrm{e}} \mathrm{s}$. (mais d'autres compléments restent possibles : là est la question, qui pour certains (tel, là...) ne tolèrent l'ordre SnomV qu'au prix d'une altération du sens de l'énoncé : la question est là):

\section{Grant cop li redona Turnus (Eneas1, ca 1155, v. 5724)}

A partir du XIV ${ }^{\mathrm{e}}$ s., et surtout du $\mathrm{XV}^{\mathrm{e}}$ s., les verbes intransitifs d'existence, de survenance, de mouvement (être, mourir, venir, ...) occupent une place croissante dans les séquences VSnom : le sujet, quel que soit son statut cognitif, est porteur dans ce cas de la charge informationnelle la plus élevée, celle du verbe étant relativement faible, en particulier pour le verbe être.

Lahousse (2011) a proposé que la caractéristique commune aux différentes structures VSnom en français moderne est le fait que le sujet n'est pas le topique principal de la phrase, soit que l'élément initial constitue un topique scénique ou bien un focus restrictif (adverbe de manière et attribut), soit, et je dirais plus souvent, que le sujet constitue un focus. Cette hypothèse est à même de rendre compte de bon nombre des postpositions nominales des siècles passés. Toutefois, comme pour VSp, et peut-être plus souvent, au moins jusqu'au $\mathrm{XV}^{\mathrm{e}}$ s., on peine à identifier dans certaines occurrences un effet focalisant, que ce soit sur l'objet ou sur le sujet.

Les effets de sens qui ont accompagné VSnom et VSp au fil des siècles relèvent du domaine pragmatique : ils sont d'ordre logico-pragmatiques pour VSp, et d'ordre pragmatico-informationnels pour VSnom.

\footnotetext{
${ }^{16} \mathrm{Ne}$ serait-ce que du fait que leur position avant le verbe est très tôt quantitativement minoritaire.
} 


\subsection{La reconfiguration d'un réseau de constructions}

La section précédente a mis en évidence la présence, dès l'ancien français, des effets de sens qui accompagnent VSnom et VSp, même si une évolution s'est produite pour VSp depuis des effets d'opposition assez diversifiés vers l'effet plus spécifique d'une validation problématique de la relation prédicative. Mais ces effets de sens ne sont pas systématiques, au moins durant la période médiévale : certains exemples résistent à une analyse en termes de focus ou d'opposition. On a donc d'un côté des constructions SV sans effets de sens spécifiques autres que le sens hérité de la macro-construction [sujet-verbe], «non marquées», et de l'autre des constructions VSnom et VSp, parmi lesquelles on trouve des occurrences pareillement non marquées, tandis que d'autres présentent un effet de sens de focalisation ou d'opposition ou de mise en balance de la prédication. Les premières vont progressivement disparaître, les constructions VSnom et VSp n'étant plus qu'associées aux effets de sens mentionnés, spécialisation du «sens» qui s'est accompagnée d'une série de restrictions sur le plan formel. On a donc désormais un système strictement structuré, et sans intersections, avec d'un côté SV et de l'autre VSnom et VSp.

Cette étude a laissé de côté des aspects importants, en particulier la question des subordonnées, et des constructions impersonnelles, de même que la présence de certains éléments initiaux devant VSp. Elle n'a tracé que les grandes lignes d'un possible cadre d'analyse de l'évolution de la position du sujet, et plus spécifiquement du recul de sa postposition. Il reste à analyser plus en détail, y compris d'un point de vue chronologique, l'évolution des propriétés des différentes micro-constructions.

\section{Références linguistiques}

Antoine G. (1958-1962), La coordination en français, Paris, Editions d'Artrey.

ARIEL M. (1988), "Referring and accessibility", Journal of Linguistics 24, 65-87.

COMBETtes B. (1988), Recherche sur l'ordre des éléments de la phrase en moyen français. Thèse pour le Doctorat d'Etat. Université de Nancy.

BEAULIER F. (1956), «Contribution à l'étude de l'inversion du sujet après la conjonction et », Le français moderne XXIV, 249-257.

BERGH L. (1952), «Quelques réflexions sur l'inversion après la conjonction et en ancien et en moyen français », in Mélanges philologie romane offerts à M. Karl Michaëlsson, Göteborg, Bergendahls boktryck, 43-55.

FIRBAS J. (1992), Functional Sentence Perspective in Written and Spoken Communication, Cambridge, Cambridge University Press.

Foulet L. (1930 [1919]), Petite syntaxe de l'ancien français, Paris, Champion.

FOURNIER N. (1998), Grammaire du français classique, Paris, Belin.

FOURNIER N. (2001), «Expression et place des constituants dans l'énoncé en français classique: la relation sujet-verbe et la relation verbe-objet », Langue française 130, 89107. 
FuCHS C. (2006), «Locatif spatial initial et position du sujet nominal : pour une approche topologique de la construction de l'énoncé », Linguisticae Investigationes 29, 61-74.

FuCHS C. (2013), «L'inversion absolue en français : deux types d'absence à l'initiale », Analecta Romanica 80, Francfort, Klosterman, 201-217.

Givón T. (1979), On Understanding Grammar, New York, Academic Press.

Guimier C. (1997), « La place du sujet clitique dans les énoncés avec adverbe initial », in C. Fuchs (éd.), La place du sujet en français contemporain, Louvain-la-Neuve, Duculot, 43 96.

LAHOUSSE K. (2011), Quand passent les cigognes. Le sujet nominal postverbal en français moderne, Paris, Presses universitaires de Vincennes.

LAMBReCht K. (1994), Information Structure and Sentence Form. Topic, Focus and the Mental Representations of Discourse Referents, Cambridge, Cambridge University Press

LEGALLOIS D. (2016). La notion de construction. In Encyclopédie grammaticale du français. En ligne: encyclogram.fr

Lehmann C. (1995 [1982]), Thoughts on Grammaticalization, Munich / Newcastle, Lincom Europa.

MARANDIN J.- M. (2003), «Inversion du sujet et discours dans les langues romanes », in. D. Godard (éd.), Les langues romanes. Problèmes de la phrase simple, Paris, Editions du CNRS, 345-392.

MarChello-Nizia C. (1985), Dire le vrai. L'adverbe si en français médiéval. Essai de linguistique historique, Genève, Droz.

MARChELlo-Nizia C. (1995), L'évolution du français. Ordre des mots, démonstratifs, accent tonique, Paris, Armand Colin.

MARChELlo-Nizia C. 1997 [1979]), La langue française aux XIV et XV siècles, Paris, Nathan

Marchello-Nizia C. (2020a), «L'objet», in C. Marchello-Nizia \& al. (éds.), Grande Grammaire Historique du Français, Berlin, De Gruyter Mouton, 1126-1156

Marchello-Nizia C. (2020b), «L'attribut», in C. Marchello-Nizia \& al. (éds.), Grande Grammaire Historique du Français, Berlin, De Gruyter Mouton, 1156-1172.

PRevost S. (2001), La postposition du sujet en français aux $15^{e}$ et $16^{e}$ siècles. Analyse sémantico-pragmatique, Paris, Editions du CNRS.

Prevost S. (2010), «Evolution de la position du sujet pronominal en français médiéval : une approche sémantico-pragmatique », in In F. Neveu \& al. (éds.), Actes du CMLF 2010-2 $2^{e}$ Congrès Mondial de Linguistique Française. Paris : Institut de Linguistique Française, 305-320 [en ligne : https://doi.org/10.1051/cmlf/2010106].

Prevost S. (2017), «Evolution de l'ordre des mots en français : grammaticalisation et rôle de la norme », in M. Velinova (éd.), Normes et grammaticalisation : le cas des langues romanes, Actes du CIER 2015, Sofia, CU Romanistika, 349-370.

Prevost S. (2020a), «Le maintien de OSV et de VOS en français moderne : un cas de rémanence? », Le Français Moderne 2020/2, 261-270.

Prevost S. (2020b), «L'ordre des constituants majeurs », in C. Marchello-Nizia \& al. (éds.), Grande Grammaire Historique du Français, Berlin, De Gruyter Mouton, 1184-1205.

Prevost S. (2020c), «Pronoms personnels et réfléchis (morpho-syntaxe)», in C. MarchelloNizia \& al. (éds.), Grande Grammaire Historique du Français, Berlin, De Gruyter Mouton,1001-1005.

Prevost S. \& Marchello-Nizia C. (2020), « Le sujet». in C. Marchello-Nizia \& al. (éds.), Grande Grammaire Historique du Français, Berlin, De Gruyter Mouton, 1055-1126.

SCHøSLER L. (1984), La déclinaison bicasuelle de l'ancien français, son rôle dans la syntaxe de la phrase, les causes de sa disparition. Etudes romanes de l'Université d'Odense, vol 19, Odense, Odense University Press. 
SKÅRUP P. (1975, Les premières zones de la proposition en ancien français. Essai de syntaxe de position. Etudes romanes de l'université de Copenhague, Revue Romane numéro spécial 6, Copenhague, Akademisk Forlag.

SMirnova E. (2015), "Constructionalization and contructional change: The role of context in the development of constructions", in J. Barðdal \& al. (éds.), Diachronic Construction Grammar, Amsterdam/Philadelphia, John Benjamins, 81-106.

Traugott E. C. \& Trousdale G. (2013), Constructionalization and Constructional Changes, Oxford, Oxford University Press.

VAnce B. (1997), Syntactic Change in Medieval French. Verb-Second and Null Subjects, Dordrecht / Boston / Londres, Kluwer Academic Publishers.

ZINK G. (1997), Morphosyntaxe du pronom personnel (non réfléchi) en moyen français (14 ${ }^{e}$ $15^{e}$ siècles), Genève, Droz.

\section{Références des textes du corpus}

Les références complètes se trouvent dans les bases dont proviennent les textes :

BFM - Base de Français Médiéval (2012-), Lyon, UMR ICAR (CNRS \& ENS de Lyon), http://bfm.ens-lyon.fr. (BFM)

Bibliothèques virtuelles humanistes http://www.bvh.univ-tours.fr, 2009 (BVH).

FRANTEXT - Base textuelle Frantext, ATILF (CNRS \& Université de Lorraine), http://www.frantext.fr. (Fr)

[ArchierBaignollet] Franc (Le) archier de Baignollet (Fr)

[Aucassin] Aucassin et Nicolette (BFM)

[BeroladeParvenir] Béroalde de Verville, François, Le Moyen de parvenir (Fr)

[BeroulTristan] Beroul, Tristan (BFM)

[CalvinLettres] Calvin, Jean, Lettres à Monsieur et Madame de Falais (Fr)

[CentNouvelles] Cent nouvelles nouvelles (Fr)

[ClariConstantinople] Robert de Clari, Conquête de Constantinople (BFM)

[Commynes] Philippe de Commynes, Mémoires (BFM)

[Descartes] Descartes, René, Discours de la méthode (Fr)

[Eneas] Eneas 1 et 2 (BFM)

[GersonSermon] Gerson, Jean, Sermon pour la fête de la Sainte Trinité. (Fr).

[Graal] Queste del saint Graal (BFM)

[Griseldis] Estoire de Griseldis en rimes et par personnages (BFM)

[JehanParis] Roman de Jehan de Paris (BFM)

[Joinville] Jean de Joinville, Vie de saint Louis $(\mathrm{Fr})$

[Lapidaire] Lapidaire en prose (BFM)

[LéryBrésil] Léry, Jean de, Histoire d'un voyage fait en la terre du Bresil. (BVH)

[MachautFortune] Guillaume de Machaut, Remede de Fortune (BFM)

[Manières] Manières de langage (BFM)

[Mesnagier] Mesnagier de Paris (BFM)

[Montaigne] Montaigne, Michel de, Essais (Fr)

[OrleansBallades] Charles d'Orléans, Ballades (BFM)

[Passion] Passion de Jésus-Christ ou Passion de Clermont (BFM)

[Pathelin] La Farce de maître Pierre Pathelin (BFM)

[QuinzeJoies] Quinze joies de mariage (BFM)

[RabutinLettres] Bussy-Rabutin, Roger de, Les Lettres de messire Roger de Rabutin, comte de Bussy (Fr)

[RenartDole] Jean Renart, Roman de la Rose ou de Guillaume de Dole (BFM).

[Roland] Chanson de Roland (BFM) 
[RonsardMisères] Ronsard, Pierre de, Discours des Miseres de ce temps (BVH) [SorelBerger] Sorel Charles, Le Berger extravagant $(\mathrm{Fr})$

[StAlexis]Vie de saint Alexis (BFM)

[StLegier]Vie de saint Léger (BFM)

[TroyesYvain] Chrétien de Troyes, Chevalier au Lion ou Yvain (BFM).

[Vigneulles] Vigneulles, Philippe de, Les Cent Nouvelles nouvelles (Fr) 